\title{
DATOS PARA LA APRECIACIÓN DE LA FLORA FANEROGÁMICA DEL BOSQUE TROPICAL CADUCIFOLIO DE MÉXICO
}

\author{
JeRzy Rzedowski ${ }^{1}$ y GRaciela CaLderón De Rzedowski \\ Instituto de Ecología, A.C., Centro Regional del Bajío, \\ Apdo. postal 386, 61600 Pátzcuaro, Michoacán, México. \\ 1Autor para la correspondencia: jerzy.rzedowski@inecol.edu.mx
}

\section{RESUMEN}

Se integra una enumeración de 749 géneros de fanerógamas que contienen al menos una especie que habita exclusiva o preferentemente en el bosque tropical caducifolio (btc) de México. Tal lista indica que la familia Leguminosae es la mejor representada en este tipo de vegetación; le siguen en importancia las Compositae y Gramineae y en seguida Malvaceae, Orchidaceae y Apocynaceae. La composición florística del btc de nuestro país con frecuencia discrepa mucho de una localidad a otra. De acuerdo con lo encontrado en la literatura, al menos 96 géneros de árboles se registran con especies dominantes o cuantitativamente importantes en los censos realizados en diferentes partes del país. Las afinidades geográficas de la flora del btc indican estrechos vínculos con las de Centro y Sudamérica. De acuerdo con este inventario, 7.9\% de los géneros son endémicos a los límites políticos de la República y esta cifra sube a $19.2 \%$ si se toma como referencia también a Centroamérica. La existencia del btc en México data al menos desde Cenozoico Temprano y su flora actual, sin menoscabo de haberse nutrido mediante intercambios de fuentes diversas, es primordialmente el resultado de largos e intensos procesos evolutivos realizados en su propio seno.

Palabras clave: bosque tropical caducifolio, flora, México.

\begin{abstract}
A list of 749 genera of flowering plants containing at least one species that grows exclusively or preferentially in the tropical deciduous forest (tdf) of Mexico is provided. The Leguminosae is the best represented family, followed by Compositae and Gramineae, Malvaceae, Orchidaceae and Apocynaceae. The floristic composition of Mexican tdf often
\end{abstract}


differs greatly from one locality to another. According to the literature, at least 96 tree genera are reported with species that are dominant or quantitatively important in inventories made in different parts of the country. The geographical affinities of the flora of tdf indicate strong relationships to those of Central and South America. 7.9\% of the genera are endemic to the political limits of Mexico and this number rises to $19.2 \%$ if Central America is also included as reference. The existence of tdf of Mexico dates at least from Early Cenozoic and its present flora, although furnished by exchanges from different sources, is primarily the result of long and intense evolutionary processes within itself.

Key words: flora, Mexico, tropical deciduous forest.

\section{INTRODUCCIÓN}

Uno de los elementos prominentes de la riqueza biológica del territorio de la República Mexicana es el bosque tropical caducifolio (btc), también con frecuencia llamado selva baja decidua o selva baja caducifolia.

El concepto de btc corresponde al conjunto de comunidades vegetales forestales de estatura baja que prosperan sobre laderas de cerros en condiciones de clima cálido pero con lluvia marcadamente concentrada en 6 o menos meses al año y en consecuencia con dos aspectos estacionales muy diferentes.

Su relevancia deriva de varias circunstancias concurrentes:

a) Cabe estimar que en condiciones prístinas el btc cubría aproximadamente $14 \%$ de la superficie del país, o sea cerca de $280,000 \mathrm{~km}^{2}$ y se le encuentra presente en 25 de las 32 entidades federativas del mismo;

b) Es el tipo de vegetación mexicano en que es más drástica la diferencia entre sus dos facetas; la verde tierna y exuberante de la temporada de lluvias y la gris y desolada de la época seca del año, contraste que implica un conjunto de notables aspectos adaptativos de su biota;

c) En lo que concierne a nuestro continente, esta comunidad vegetal existe también en Centro y Sudamérica, así como en varias islas antillanas, pero es en México donde encuentra mucho más amplia representación, variedad, así como magnitud de endemismo.

Los primeros en estudiar la composición florística del btc en México fueron Shreve (1937) en Baja California Sur, Miranda (1941, 1942, 1943, 1947, 1948) en la Depresión del Balsas y en la parte alta de la Cuenca del Papaloapan, H. S. Gentry (1942, 1946) en Sonora y Sinaloa, así como Leavenworth (1946) en Michoacán. 
En la actualidad se cuenta ya con numerosos inventarios de las plantas que integran este tipo de vegetación de muy diversas localidades del país y partiendo de tal base surgió la idea de realizar esta sinopsis preliminar. En esencia el trabajo consiste en la elaboración de un censo de los géneros de fanerógamas que incluyen al menos una especie que crece de manera exclusiva o preferencial en el btc de México, y consecuentemente de algunos análisis basados en esta enumeración.

\section{MÉTODO}

La integración de la lista de géneros se realizó primordialmente mediante la búsqueda bibliográfica. Se consultaron más de 60 inventarios de plantas del btc de diferentes comarcas del país y para localizarlos fue de gran ayuda el compendio de Guízar Nolasco (2000).

Se revisaron asimismo varias floras y listas florísticas y de particular utilidad resultaron ser los tomos publicados de Flora Novo-Galiciana de McVaugh y colaboradores (1983-2001).

Para algunos grupos de plantas se recurrió a estudios monográficos y de revisión taxonómica, en particular a los más modernos, que con frecuencia son más ricos en la información ambiental de las especies, como p. e. la obra de Fryxell (1988).

La selección de los géneros se basó en gran medida en la interpretación de la información disponible sobre la distribución geográfica y ecológica de las especies, pero dado el hecho de que ésta no siempre es clara, completa y totalmente fidedigna, no hay duda de la existencia de un importante margen de error. Los autores consideran, sin embargo, que este último no alcanza llegar a $10 \%$ y muy probablemente deben prevalecer allí los nombres faltantes y no los sobrantes.

En total se hizo uso de datos encontrados en alrededor de 145 contribuciones, que incluyen numerosos trabajos de tesis y algunos manuscritos que tampoco se han publicado formalmente.

Además, en repetidos casos de duda se efectuó la consulta directa de ejemplares de herbario y se acudió a la experiencia personal de los autores, así como de varios botánicos especialistas, cuyos nombres aparecen al final en el apartado de los agradecimientos.

Se ha procurado no considerar en esta integración a las plantas que aun siendo bastante características del ambiente del btc, se observan mayormente en calidad de malezas ni tampoco las introducidas de otros continentes. En cambio sí se inclu- 
yeron varias que, sin ser propiamente habitantes del bosque, prosperan en algunos microambientes adyacentes al mismo.

En términos generales se ha tratado de aceptar las recientes modificaciones taxonómicas surgidas primordialmente a raíz de estudios realizados con ayuda de secuencias de ADN. Sin embargo, siguiendo a Fryxell (2001), se ha preferido mantener como familias independientes a Bombacaceae, Sterculiaceae y Tiliaceae.

De este modo se logró reunir un conjunto de 749 géneros que, sin pretender ser perfecto, cabe considerar como representativo de la flora del btc de nuestro país y de las comunidades secundarias derivadas del mismo.

\section{EL BOSQUE TROPICAL CADUCIFOLIO DE MÉXICO}

Los datos más esenciales relativos a este tipo de vegetación pueden consultarse en Rzedowski (1978: 189-203). Sin embargo, cabe enmendar algunos aspectos, ya que hallazgos posteriores a esta fecha (Rzedowski y Calderón, 1987) han confirmado la presencia del btc (en la actualidad casi totalmente sustituido por comunidades secundarias) en las porciones cerriles de la región del Bajío, donde su área llega hasta los alrededores de las ciudades de Guanajuato, Morelia y Querétaro. Entre otros lugares se encontró la existencia de sus vestigios al norte de la región urbana de León, donde alcanza altitudes del orden de 2,300 m.

Tal información define el hecho, también confirmado por Trejo Vázquez (1998, 1999), de que los btc, al menos algunas de sus modalidades y componentes, son capaces de tolerar también la incidencia repetitiva de heladas durante todos o casi todos los años, característica desconocida con anterioridad.

En los mencionados trabajos de Trejo Vázquez $(1998,1999)$ cabe encontrar asimismo muy significativos pormenores relativos al ambiente, así como a la diversidad de la flora leñosa del btc de diferentes regiones de México y las relaciones geográficas de la misma.

El mapa de la Fig. 1 ilustra una representación esquemática de la distribución de este tipo de vegetación en el territorio de la República Mexicana, pero corresponde más bien a su área potencial que real, pues más de tres cuartas partes de la misma se encuentran hoy convertidas en diferentes tipos de comunidades secundarias, así como en parcelas agrícolas, hortícolas y potreros, sin menoscabo de muy numerosos desarrollos urbanos, industriales y turísticos, de pequeñas y grandes represas, vías de comunicación y ductos de diferentes clases. 


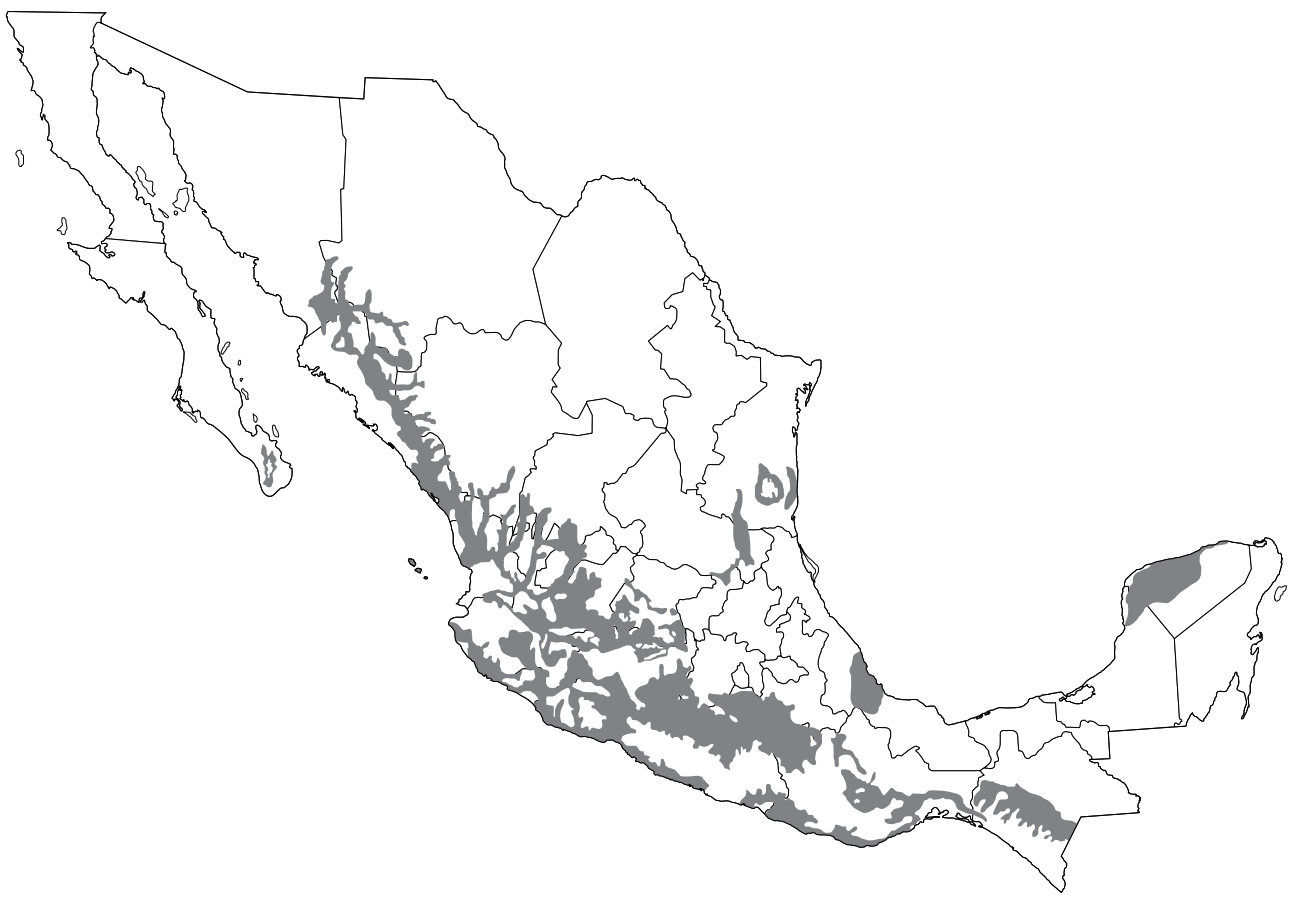

Fig. 1. Mapa esquemático de la distribución geográfica del bosque tropical caducifolio en México.

Es tan grande la magnitud de esta desforestación que, como lo indicaron varios autores (Rzedowski y Calderón, 1987; Trejo y Dirzo, 2000, 2002), algunos sectores del país ya han perdido casi por completo la cobertura original del btc. Este es el caso de la región del Bajío y de áreas aledañas de Jalisco, de la parte central de Veracruz y de la Depresión Central de Chiapas. La Huasteca potosina y tamaulipeca se encuentra también cercana a la misma situación.

Independientemente de los efectos de la actual intensa expansión demográfica de la población de nuestro país, existen tres factores de gran peso que han estado y siguen contribuyendo a la rápida desaparición de este bosque, a mencionar:

a) el desfavorable hecho de que el btc es en general una comunidad biótica particularmente lábil, pues se desnaturaliza con gran facilidad y carece de buena capacidad de rápida recuperación, pues las asociaciones vegetales que lo sustituyen con frecuencia (sobre todo con la ayuda del hombre) son más estables que el bosque clímax; 
b) la otra poco propicia circunstancia es que por lo general las plantas del btc son de relativamente escasa importancia económica para la población humana, por lo que ésta a menudo busca cambiarlo por elementos de mayor posibilidad de aprovechamiento;

c) el tercer factor, de especial significación en México, es la particularidad de que el clima de los lugares en que habitan los btc, sobre todo de los ubicados a altitudes superiores a 1,200 $\mathrm{m}$, es de gran atractivo para el hombre, tanto para su comodidad, bienestar y salud, como por el extenso abanico de cultivos agrícolas y plantaciones hortícolas permanentes que permite; a su vez, muchos sectores cercanos al mar resultan ser ideales para grandes desarrollos turísticos.

\section{COMPOSICIÓN FLORÍSTICA}

Los btc de diferentes partes de México se parecen relativamente poco entre sí en términos de su flora, por lo que la expresión global de esta última es vasta y puede estimarse en el orden de 4,500 a 5,000 especies, si se incluyen también las comunidades secundarias derivadas de los mismos.

Por otra parte, sin embargo, una gran proporción de sus componentes se comparten con otros tipos de vegetación, a menudo adyacentes, como el bosque espinoso, el tropical subcaducifolio, así como con matorrales xerófilos, pastizales y encinares. De este modo, la cantidad de elementos que pertenecen en forma exclusiva o preferencial al btc sólo debe alcanzar poco más de la mitad, o sea cerca de 2,500 especies.

Infortunadamente la información existente no es suficiente para aventurar un inventario realista de este conjunto de plantas, pero a título de aproximación se presenta aquí un intento de censar 749 géneros que contienen al menos una especie con las mencionadas características, cuya lista aparece en el Apéndice de esta contribución y que constituye la base del texto que se está formulando en seguida. Tal cifra representa más de la cuarta parte de los géneros de fanerógamas conocidos como integrantes de la flora de México.

El Cuadro 1 enumera las 14 familias mejor representadas en la flora del btc de México, que en su conjunto agrupan 446 géneros, o sea aproximadamente $60 \%$ del total registrado de los 749 .

Acerca de la preponderancia de Leguminosae en el btc ya se sabía desde hace mucho, por lo que no constituye ninguna sorpresa. La gran abundancia de los com- 
Cuadro 1. Familias mejor representadas en la flora del btc de México, indicando su contenido de géneros que se caracterizan por comprender al menos una especie que se distribuye en forma exclusiva o preferencial en este tipo de vegetación.

\begin{tabular}{lclc}
\hline Familia & Núm. de géneros & Familia & Núm. de géneros \\
\hline Leguminosae & 85 & Rubiaceae & 21 \\
Compositae & 78 & Acanthaceae & 20 \\
Gramineae & 41 & Cucurbitaceae & 19 \\
Malvaceae & 31 & Bignoniaceae & 18 \\
Orchidaceae & 30 & Malpighiaceae & 18 \\
Apocynaceae & 30 & Euphorbiaceae & 17 \\
Cactaceae & 24 & Sapindaceae & 13 \\
\hline
\end{tabular}

ponentes de Compositae y Gramineae igualmente era de esperarse, pero lo que es más revelador es la poco conocida importancia de Orchidaceae, Malvaceae y Apocynaceae (sensu lato), así como de varias familias representadas total o ampliamente por plantas trepadoras, como son las Apocynaceae, Bignoniaceae, Cucurbitaceae, Malpighiaceae y Sapindaceae.

Respecto a la ubicación ecológica, cabe comentar que la trascendencia de Compositae y Gramineae se observa con frecuencia sólo en los matorrales y pastizales secundarios derivados del btc. Las Leguminosae son muy prominentes tanto en las comunidades clímax, como en las sucesionales.

Entre las Orchidaceae son relativamente pocas las terrestres y epipétricas, predominando manifiestamente las epífitas, por lo que su incidencia por lo general está correlacionada con las condiciones de una favorable humedad atmosférica y temperatura a lo largo del año. Como ya lo han señalado Hágsater et al. (2005), las orquídeas faltan por completo en muchos btc de México, sobre todo en los ubicados en el norte de la República, así como a mayores altitudes. Tampoco son comunes en las fases secundarias del bosque y en general son muy sensibles a la perturbación.

A su vez, la abundancia de los representantes de Cactaceae está en general claramente vinculada con las facetas más secas del btc, y en particular la de las especies de Opuntia con el disturbio.

Otro hecho interesante es que un importante conjunto de familias de la flora fanerogámica de México concentra la presencia de sus representantes en este tipo de vegetación. Entre las enumeradas en el Cuadro 1 calificarían aquí Leguminosae, Malvaceae, Acanthaceae, Cucurbitaceae, Malpighiaceae, Euphorbiaceae y Sapindaceae. 
En términos de grandes ausencias, cabe señalar en primer lugar la falta casi completa de las gimnospermas, pues de este grupo sólo se registra la poco trascendente presencia de Dioon.

De las familias de angiospermas ampliamente representadas en la flora de México cabe observar que entre los géneros con al menos una especie exclusiva o preferente del btc al parecer no se encuentra ninguno perteneciente a las siguientes:

Chenopodiaceae

Cruciferae

Ericaceae
Fagaceae

Melastomataceae

Myrsinaceae

sin que esto signifique que tales familias necesariamente carezcan por completo de representantes ocasionales o de amplia tolerancia ecológica en el tipo de vegetación en cuestión.

También es interesante constatar que la riqueza y la heterogeneidad de la flora (diversidades alfa y beta) aumentan en general de manera gradual de norte a sur de México e igualmente de este a oeste, alcanzando sus expresiones máximas en la Depresión del Balsas y en la región costera entre Michoacán y el Istmo de Tehuantepec. Este hecho ya quedó puesto de manifiesto para los árboles del btc mediante los valores numéricos dados a conocer por Trejo y Dirzo (2002), pero no hay mucha duda de que cabe extrapolarlo para la totalidad de flora de esta comunidad vegetal.

Cuadro 2. Géneros con mayor representación de especies que se distribuyen en forma exclusiva o preferencial en el btc de México.

\begin{tabular}{lllll}
\hline Abutilon & Colubrina & Heliotropium & Marsdenia & Serjania \\
Acacia & Cordia & Indigofera & Matelea & Sida \\
Acalypha & Croton & Ipomoea & Mimosa & Sideroxylon \\
Aristolochia & Cuphea & Jatropha & Opuntia & Stenocereus \\
Ayenia & Desmodium & Justicia & Otopappus & Tillandsia \\
Brongniartia & Dioscorea & Leucaena & Passiflora & Triumfetta \\
Bursera & Diospyros & Lonchocarpus & Perymenium & Trixis \\
Caesalpinia & Euphorbia & Lysiloma & Randia & Verbesina \\
Calliandra & Gonolobus & Manihot & Ruellia & Vernonia \\
Cephalocereus & Heliocarpus & Marina & Senna & \\
\hline
\end{tabular}


En el Cuadro 2 se enumeran 49 géneros que, de acuerdo con la literatura y los herbarios consultados, son los que están representados en el btc de México por un mayor número de especies que se distribuyen en forma exclusiva o preferencial en el mismo.

En este conjunto de nuevo predominan las Leguminosae (con 12 componentes), seguidas por Compositae (con 5 géneros). Sin embargo, faltan por completo las Gramineae y las Orchidaceae, pero en cambio aparecen miembros de otras familias que, si bien no registran muchos géneros, si marcan su presencia por un significativo número de especies. Estas son: Aristolochiaceae, Boraginaceae, Bromeliaceae, Burseraceae, Convolvulaceae, Dioscoreaceae, Lythraceae, Passifloraceae y Tiliaceae. Descuellan sobre todo Bursera (Burseraceae), Euphorbia (Euphorbiaceae), Ipomoea (Convolvulaceae) y Mimosa (Leguminosae), cada una con más de 50 especies.

El Cuadro 3 integra una lista (indudablemente incompleta) de 96 géneros cuyas especies de árboles se registran como dominantes o cuantitativamente importantes en la biomasa del btc de muchas localidades de México. La magnitud de esta enumeración es una evidente muestra de la baja similitud florística que a menudo tienen entre sí los btc no sólo de diferentes regiones del país, sino también con frecuencia de distintos rodales de una misma comarca.

\section{AFINIDADES GEOGRÁFICAS DE LA FLORA}

Trejo Vázquez (1998) realizó ya un examen inicial de las afinidades geográficas de la flora del btc de México, basado en 917 especies, pertenecientes 368 géneros de árboles y arbustos inventariados en diferentes regiones del país. De tal modo el ejercicio que a continuación se expone cabe considerarlo como complementario del estudio mencionado, al que a continuación se hará referencia como TV98.

El Cuadro 4 define el análisis de la distribución geográfica total de los 749 géneros de fanerógamas reconocidos en este trabajo como representativos del btc en el territorio de la República. Las proporciones encontradas confirman en primera instancia el conocido hecho de que la flora del btc de México sólo tiene muy escasas ligas con el norte y se encuentra preponderantemente vinculada con la de Centro y Sudamérica. Para enfatizar esta relación cabe señalar que la mayor parte de los 25 géneros que extienden su área de distribución de México a Estados Unidos, no penetra profundamente al interior de ese último país y su área corresponde a la denominada por Rzedowski (1991) como Megaméxico 1. Este aspecto coincide bien con lo encontrado por TV98. 
Cuadro 3. Géneros con al menos una especie arbórea registrada en la literatura como dominante o cuantitativamente importante en el btc de México o en alguna de sus comunidades sucesionales.

\begin{tabular}{|c|c|c|}
\hline Acacia & Erythrina & Pachycereus \\
\hline Actinocheita & Erythroxylon & Parmentiera \\
\hline Achatocarpus & Escontria & Pilosocereus \\
\hline Agonandra & Esenbeckia & Piptadenia \\
\hline Albizia & Eugenia & Piscidia \\
\hline Alvaradoa & Euphorbia & Pistacia \\
\hline Amphipterygium & Eysenhardtia & Pithecellobium \\
\hline Apoplanesia & Ficus & Plumeria \\
\hline Beaucarnea & Fouquieria & Pseudobombax \\
\hline Bernardia & Fraxinus & Pseudosmodingium \\
\hline Brahea & Gossypium & Psidium \\
\hline Brongniartia & Guaiacum & Randia \\
\hline Bunchosia & Guazuma & Sabal \\
\hline Bursera & Gymnopodium & Savia \\
\hline Caesalpinia & Gyrocarpus & Senna \\
\hline Capparis & Haematoxylon & Sideroxylon \\
\hline Cedrela & Hampea & Simarouba \\
\hline Ceiba & Hauya & Spondias \\
\hline Cephalocereus & Havardia & Stemmadenia \\
\hline Cercidium & Helicteres & Stenocereus \\
\hline Cnidoscolus & Heliocarpus & Swietenia \\
\hline Coccoloba & Hintonia & Tabebuia \\
\hline Cochlospermum & Ipomoea & Tecoma \\
\hline Colubrina & Jacaratia & Terminalia \\
\hline Comocladia & Jatropha & Thevetia \\
\hline Conzattia & Leucaena & Thouinia \\
\hline Cordia & Lonchocarpus & Trichilia \\
\hline Croton & Lysiloma & Viguiera \\
\hline Cyrtocarpa & Mimosa & Vitex \\
\hline Dalbergia & Neobuxbaumia & Wimmeria \\
\hline Diospyros & Neomillspaughia & Ziziphus \\
\hline Ehretia & Ocotea & Zuelania \\
\hline
\end{tabular}


Cuadro 4. Análisis de la distribución geográfica total de los 749 géneros de fanerógamas enumerados en el Apéndice.

\begin{tabular}{|c|c|c|c|}
\hline Tipo de distribución & & Núm. de géneros & Porcentaje del total \\
\hline Cosmopolita o cercana a cosmopolita & & 39 & 5.2 \\
\hline Pantropical o cercana a pantropical & & 141 & 19.0 \\
\hline América & & 35 & 4.7 \\
\hline México a Sudamérica & & 243 & 32.4 \\
\hline América tropical y África & & 28 & 3.7 \\
\hline Hemisferio norte & & 3 & 0.4 \\
\hline México y Centroamérica & & 85 & 11.3 \\
\hline México y Estados Unidos & & 25 & 3.3 \\
\hline México y las Antillas & & 3 & 0.4 \\
\hline México & & 59 & 7.9 \\
\hline \multirow[t]{2}{*}{ Otros tipos de distribución } & & 88 & 11.7 \\
\hline & Total & 749 & 100 \\
\hline
\end{tabular}

Por otro lado es interesante comentar que de los 243 géneros comunes con la flora de Sudamérica, más de la mitad existe también en las Antillas. Sin embargo, los compartidos sólo por México y las islas del Caribe son únicamente tres, circunstancia que indica una más bien escasa vinculación directa con la flora de este archipiélago, igualmente encontrada por TV98.

Con África tampoco existe una relación cercana. No obstante, llama la atención la presencia de 28 géneros comunes entre América tropical y ese continente, situación que en gran medida procede atribuir al hecho de que en grandes extensiones de África prevalece un clima cálido estacional de larga temporada de sequía.

En cuanto al endemismo, TV98 encontró que $5.4 \%$ de géneros $60 \%$ de las especies registran una distribución restringida a los límites políticos de México. Descubrió asimismo que las proporciones de estos elementos varían muy notablemente de un lugar a otro y así, a nivel de género, entre los sitios inventariados descollaron por sus valores elevados (16.2 a 23.1\%) dos de la cuenca alta del Papaloapan y uno de la Depresión del Balsas, mientras que el de Tamaulipas y el de Yucatán carecieron por completo de estas plantas.

El presente inventario reveló la presencia de una significativamente mayor (7.9\%) proporción de géneros de distribución restringida a México que la encon- 
trada por TV98. Tal discrepancia es interesante e indica que el endemismo a este nivel taxonómico está más pronunciado en el sotobosque, en la sinusia epifítica y probablemente en los claros en medio del btc que en el dosel arbóreo del mismo. Los miembros de las familias Compositae, Acanthaceae, Alliaceae, Cucurbitaceae, Crassulaceae, Orchidaceae, Malvaceae y Malpighiaceae son los principales responsables de esta desigualdad numérica.

De los 59 géneros de distribución restringida a los límites de México, 26 están constituidos por una sola especie y de éstos nueve únicamente se conocen de la localidad tipo o también de algunos lugares aledaños.

Para el género Bursera, que es uno de los más característicos componentes del btc mexicano pueden ofrecerse los datos numéricos del Cuadro 5.

Cuadro 5. Algunos datos numéricos relativos a las especies mexicanas del género Bursera.

Total de especies conocidas de México

Número estimado de especies adicionales, aún por describirse o definirse aprox. 20

Número de especies conocidas endémicas a los límites de México

Número de especies solo conocidas de uno o dos estados contiguos de la

República

Número de especies solo conocidas de la localidad tipo y de lugares aledaños

Número de especies de distribución restringida a la Depresión del Balsas

Número de especies que prosperan en forma exclusiva o preferencial en el btc

En términos de mayores disyunciones cabe apuntar cerca de dos decenas de géneros representados en México y en Sudamérica, sin que se conozca su presencia en el istmo centroamericano ni en las Antillas. Entre estas quizás el más notable es el caso de Lennoa, pequeño parásito de raíces de otras plantas herbáceas, relativamente bien distribuido en México, con penetración a Guatemala, y registrado también de la región costera atlántica de Colombia y Venezuela.

En resumen, la flora del btc de México es de carácter esencialmente neotropical, con una cuantiosa participación de elementos locales, probablemente en su mayoría autóctonos, tanto a nivel de especie, como también de género y aun de familia, pues una de ellas (Pterostemonaceae) se registra exclusiva de los límites de nuestro país y dos (Fouquieriaceae y Plocospermataceae) no exceden las fronteras de Megaméxico. 


\section{ORÍGENES DE LA FLORA DEL BTC DE MÉXICO}

Existen numerosos indicios de que el btc tiene una larga historia en nuestro país y en tal sentido se pronuncian tanto la riqueza como la distribución heterogénea de su flora, al igual que la considerable magnitud de su endemismo, hechos que ya se conocían desde hace algún tiempo.

Más recientemente, mediante estudios de desenvolvimiento filogenético y de consecuente "reloj molecular", realizados con base en las secuencias de ADN de núcleo y de cloroplasto, se ha podido confirmar la existencia de este tipo de vegetación en México al menos a partir del Cenozoico Temprano (hace 50 a 60 millones de años) (de Nova et al., 2011; Becerra et al., 2012). Estas investigaciones fundamentaron sus conclusiones mediante datos derivados de la cronología evolutiva de los componentes del género Bursera, cuantioso grupo de especies muy estrechamente vinculado con el btc de toda el área mesoamericana.

En términos de orígenes, varios autores, entre otros A. H. Gentry (1995: 165, 167), postularon que la flora de los btc americanos deriva esencialmente de la de las selvas propias de ambientes cálido-húmedos del continente. Sin embargo, como ya lo puntualizó Challenger (1998: 389-390), esta procedencia sólo se manifiesta en México en una proporción limitada. Dada la ubicación geográfica de nuestro país, es muy probable que durante largas épocas y en forma bastante continua existían en él más superficies caracterizadas por la pluviosidad de tipo estacional que por las de clima francamente lluvioso. De esta manera el btc debe haber tenido suficiente tiempo para desarrollar una propia y diversa flora termófila, como lo pueden ejemplificar los numerosos endemismos, así como los cuantiosos géneros de Compositae, Malvaceae, Anacardiaceae, Acanthaceae, Rhamnaceae, Rutaceae, al igual que no pocos de las Leguminosae.

El grueso, si no la totalidad, de los representantes de Cactaceae, Agavaceae y Nolinaceae, así como de Condalia y Fouquieria del btc de México definitivamente no deriva de antecesores propios de las selvas húmedas y su origen cabe buscarlo más bien en los matorrales xerófilos. Muchos de los géneros de Compositae, al igual que Carex, Echeveria, Lamourouxia, Lobelia, Prunus, Salvia, Stachys, Thalictrum, Tigridia, Vicia y algunos otros marcan una vinculación con la flora de los encinares y bosques de coníferas de México, en cambio al menos varios de los de Gramineae provienen verosímilmente de la de los pastizales y de la vegetación sabanoide.

Si bien es evidente que las afinidades geográficas de los géneros del btc de México se dirigen en forma mayoritaria hacia el sur (Centro y Sudamérica), tal liga en buena medida se debe al hecho de que al norte de nuestro país no existen en la 
actualidad las condiciones ambientales propicias para el crecimiento de la mayoría de las plantas ecológicamente ligadas con el btc y no sería correcto deducir de allí una casi global procedencia meridional.

Aquí es apropiado hacer referencia al trabajo de Wendt (1998), quien calculó que al menos una cuarta parte de los géneros de árboles del dosel de los bosques tropicales húmedos de la vertiente atlántica de México han entrado a este país por la vía migratoria laurásica en la época (Cenozoico Temprano) cuando los climas cálidos predominaron en la mayor parte del Planeta. De manera paralela, Sousa y Delgado (1988) consideraron que una significativa fracción de los géneros mexicanos de Leguminosae usaron la misma entrada. Entre tales elementos florísticos cuentan tanto los originarios de la propia Laurasia, como también los procedentes de Gondwana, que utilizaron este camino de desplazamiento.

Es muy similar el caso del género Bursera, uno de los más característicos del btc de México, que pertenece a una familia francamente gondwaniana. Sin embargo, se sabe (Rzedowski, 1991: 17) que su distribución geográfica actual, así como la información del registro fósil, claramente sugieren que no ha arribado desde el sur.

Varios otros géneros, como Bourreria, Cordia, Ehretia, Ficus, Lonchocarpus, Nectandra, Ocotea, Sideroxylon, Zanthoxylum (Wendt, op. cit.), así como Celtis, Fraxinus, Loeselia, Pinguicula, Prunus, Rhus, Salvia, Stachys y probablemente otro cuantioso contingente más, deben también ingresado por el norte.

De esta manera cabe visualizar la flora actual del btc de México como un amalgama de elementos de procedencia y vías de entrada bastante diversas, y como un conjunto de manifiesta individualidad propia, nutrida mediante intercambios con las comunidades vegetales contiguas y sobre todo a través de largos procesos evolutivos de sus diversos componentes.

\section{AGRADECIMIENTOS}

Varios colegas tuvieron la bondad de revisar y depurar las listas de las familias de su competencia: Acanthaceae - Thomas F. Daniel; Convolvulaceae - Eleazar Carranza; Cucurbitaceae - Rafael Lira; Euphorbiaceae (sensu lato) - Victor W. Steinmann; Leguminosae - Mario Sousa, Malpighiaceae - William R. Anderson; Orchidaceae - Rolando Jiménez y Eric Hágsater; Scrophulariaceae (sensu lato) Emmanuel Pérez. Además Silvia Salas, Victor Steinmann e Irma Trejo leyeron un manuscrito preliminar y enriquecieron el texto con sus comentarios, adiciones y sustracciones. Los autores agradecen profundamente esta valiosa colaboración. 
Rosa María Murillo y Damián Piña auxiliaron en la apropiada preparación del mapa. A Georgina Ortega se le dan las gracias por la ayuda recibida para encontrar fuentes bibliográficas. El trabajo fue realizado con apoyo económico del Instituto de Ecología, A.C., del Consejo Nacional de Ciencia y Tecnología y de la Comisión Nacional para el Conocimiento y Uso de la Biodiversidad.

\section{LITERATURA CITADA}

Becerra, J. X., K. Noge, S. Olivier y D. L. Venable. 2012. The monophylly of Bursera and its impact for divergence times of Burseraceae. Taxon 61: 333-343.

Challenger, A. 1998. Utilización y conservación de los ecosistemas terrestres de México: pasado, presente y futuro. Comisión Nacional para el Conocimiento y Uso de la Biodiversidad, Universidad Nacional Autónoma de México y Agrupación Sierra Madre, S.C. México, D.F. 847 pp.

De-Nova, J. A., R. Medina, J. C. Monter, A. Weeks, J. A. Rosell, M. E. Olson, J. E. Eguiarte y S. Magallón. 2011. Insights into the construction of species-rich Mesoamerican dry tropical forests: the diversification of Bursera (Burseraceae, Sapindales). New Phytologist 193: 276-287.

Fryxell, P. A. 1988. Malvaceae of Mexico. Syst. Bot. Monogr. 25: 1-522.

Fryxell, P. A. 2001. Tiliaceae, Sterculiaceae, Bombacaceae. Flora Novo-Galiciana 3: 64-159. Gentry, A. H. 1995. Diversity and floristic composition of tropical dry forests. In. Bullock, S. H., H. A. Mooney y E. Medina. Seasonally dry tropical forests. Cambridge University Press. Cambridge. pp. 146-194.

Gentry, H. S. 1942. Rio Mayo plants. Carn. Inst. Wash. Publ. 527: 1-328.

Gentry, H. S. 1946. Sierra Tacuichamona - a Sinaloan plant locale. Bull. Torr. Bot. Club 73: 356-362.

Guízar Nolasco, E. 2000. Bibliografía sobre el bosque tropical caducifolio. Universidad Autónoma Chapingo, División de Ciencias Forestales. Bol. Técn. 32: 1- 263.

Hágsater, E., M. Soto, G. Salazar, R. Jiménez, M. López y R. Dressler. 2005. Orquídeas de México. Instituto Chinoin, A.C., México, D.F. 304 pp.

Leavenworth, W. C. 1946. A preliminary study of the vegetation of the region between Cerro Tancitaro and the Rio Tepalcatepec, Michoacan, Mexico. Amer. Midl. Natur. 36: 137-206.

McVaugh, R. et al., Flora Novo-Galiciana. 1983-2001. The University of Michigan Press y The University of Michigan Herbarium. Ann Arbor, Mich. Vols. 3, 5, 12, 13, 14, 15, $16,17$.

Miranda, F. 1941. Estudios sobre la vegetación de México. I. La vegetación de los cerros al sur de la Meseta de Anáhuac - el cuajiotal. An. Inst. Biol. Méx. 12: 569-614.

Miranda, F. 1942. Estudios sobre la vegetación de México. II. Notas generales sobre la vegetación del suroeste del estado de Puebla. An. Inst. Biol. Méx. 13: 417-450.

Miranda, F. 1943. Estudios sobre la vegetación de México. IV. Algunas características de la flora y de la vegetación de la zona de Acatlán, Puebla. An. Inst. Biol. Méx. 14: 407-421. 
Miranda, F. 1947. Estudios sobre la vegetación de México. V. Rasgos de la vegetación en la cuenca del río de las Balsas. Rev. Soc. Mex. Hist. Nat. 8: 95-114.

Miranda, F. 1948. Datos sobre la vegetación de la cuenca alta del Papaloapan. An. Inst. Biol. Méx. 19: 333-364.

Rzedowski, J. 1978. Vegetación de México. Ed. Limusa. México, D.F. 432 pp.

Rzedowski, J. 1991. Diversidad y orígenes de la flora fanerogámica de México. Acta Bot. Mex. 14: 3-21.

Rzedowski, J. y C. Calderón. 1987. El bosque tropical caducifolio en la región mexicana del Bajío. Trace 12: 12-21.

Shreve, F. 1937. The vegetation of the Cape region of Baja California. Madroño 4: 105-113.

Sousa S., M. y A. Delgado S. 1998. Leguminosas mexicanas: fitogeografía, endemismo y orígenes. In: Ramamoorthy, T. P., R. Bye, A. Lot y J. Fa (eds.). Diversidad biológica de México, orígenes y distribución. Instituto de Biología, Universidad Nacional Autónoma de México. México, D.F. pp. 449-493.

Trejo Vázquez, R. I. 1998. Distribución y diversidad de selvas bajas de México: relaciones con el clima y con el suelo. Tesis de doctorado. Facultad de Ciencias. Universidad Nacional Autónoma de México. México, D.F. 210 pp.

Trejo Vázquez, I. 1999. El clima de la selva baja caducifolia en México. Invest. Geogr. Bol. 39: 40-51.

Trejo, I. y R. Dirzo. 2000. Deforestation of seasonally dry tropical forests: a national and local analysis in Mexico. Biol. Conserv. 94: 133-142.

Trejo, I. y R. Dirzo. 2002. Floristic diversity of Mexican seasonally dry tropical forests. Biodivers. Conserv. 11: 2063-2048.

Wendt, T. 1998. Composición, afinidades florísticas y orígenes de la flora arbórea del dosel de los bosques tropicales húmedos de la vertiente mexicana del Atlántico. In: Ramamoorthy, T. P., R. Bye, A. Lot y J. Fa (eds.) Diversidad biológica de México, orígenes y distribución. Instituto de Biología, Universidad Nacional Autónoma de México. México, D.F., pp. 581-664. 
Rzedowski y Calderón de Rzedowski: Flora fanerogámica del bosque tropical caducifolio, México

\section{APÉNDICE}

Géneros de fanerógamas con al menos una especie que habita en forma exclusiva o preferencial en el bosque tropical caducifolio de México. Los marcados con asterisco $\left(^{*}\right)$ son endémicos a los límites de la República.

Acanthaceae
Anisacanthus, Aphanosperma*, Aphelandra,
Carlowrightia, Chileranthemum, Dicliptera,
Dyschoriste, Elytraria, Gypsacanthus*,
Henrya, Holographis*, Hoverdenia*,
Justicia, Louteridium, Mexacanthus*,
Odontonema, Poikilacanthus, Ruellia,
Stenandrium, Tetramerium

Achatocarpaceae

Achatocarpus

Agavaceae

Agave, Manfreda, Yucca

Alliaceae

Allium, Bessera*, Dandya*, Milla,

Petronymphe*

Amaranthaceae

Alternanthera, Amaranthus, Celosia,

Chamissoa, Gomphrena, Iresine, Lagrezia

Amaryllidaceae

Hymenocallis, Sprekelia*, Zephyranthes

Anacardiaceae

Actinocheita*, Comocladia, Cyrtocarpa*,

Pistacia, Psudosmodingium*, Rhus,

Spondias

Annonaceae

Annona, Sapranthus

Anthericaceae

Echeandia
Apocynaceae

Alstonia, Asclepias, Blepharodon,

Cynanchum, Dictyanthus, Echites, Fernaldia, Forsteronia, Funastrum, Gonolobus, Haplophyton, Laubertia, Macroscepis, Mandevilla, Marsdenia, Matelea, Metastelma, Microdactylon*, Pentalinon, Plumeria, Polystemma, Prestonia, Prosthecidiscus, Rauvolfia, Stemmadenia, Tabernaemontana, Telosiphonia, Thenardia, Thevetia, Vallesia

Araceae

Anthurium, Philodendron

Araliaceae

Aralia, Sciadodendron

Aristolochiaceae

Aristolochia

Basellaceae

Anredera

Begoniaceae

Begonia

Bignoniaceae

Adenocalymma, Amphilophium, Anaemopegma, Arrabidaea, Clytostoma, Cydista, Distictis, Godmania, Macfadyena, Mansoa, Melloa, Parmentiera, Phaedranthus*, Pithecoctenium, Stizophyllum, Tabebuia, Tecoma, Xylophragma

Bombacaceae

Ceiba, Pseudobombax 
Apéndice. Continuación.

\begin{tabular}{ll}
\hline $\begin{array}{l}\text { Boraginaceae } \\
\text { Bourreria, Cordia, Ehretia, Heliotropium, } \\
\text { Tournefortia, Varronia }\end{array}$ & $\begin{array}{l}\text { Chrysobalanaceae } \\
\text { Couepia, Licania }\end{array}$ \\
$\begin{array}{l}\text { Bromeliaceae } \\
\text { Aechmea, Billbergia, Bromelia, Catopsis, }\end{array}$ & Clusiaceae \\
Hechtia, Pitcairnia, Tillandsia, Viridantha* & Cochlospermaceae \\
& Amoreuxia, Cochlospermum \\
Burseraceae & Combretaceae \\
Beiselia*, Bursera & Combretum, Terminalia
\end{tabular}

Buxaceae

Buxus

Commelinaceae

Cactaceae

Acanthocereus, Backebergia*,

Cephalocereus, Cylindropuntia, Disocactus, Echinocereus, Escontria*, Ferocactus, Hylocereus, Mammillaria, Melocactus, Callisia, Commelina, Thyrsanthemum*, Tinantia, Tradescantia, Tripogandra

Myrtillocactus, Neobuxbaumia*, Nopalea, Opuntia, Pachycereus, Peniocereus, Pereskia, Pereskiopsis, Pilosocereus, Polaskia*, Pterocereus*, Selenicereus, Stenocereus

Campanulaceae

Diastatea, Lobelia

Capparaceae

Compositae

Acourtia, Adenophyllum, Ageratina, Ageratum, Baccharis, Baltimora, Bidens, Boeberoides*, Bolanosa*, Brickellia, Calea, Carminatia, Chromolaena, Chrysanthellum, Coreocarpus*, Cosmos, Critonia, Decachaeta, Delilia, Dyssodia, Eryngiophyllum*, Espejoa, Eupatoriastrum, Flaveria, Fleischmannia, Florestina, Flourensia, Flyrella, Galeana, Galinsoga, Gochnatia, Grindelia, Guardiola, Gymnolaena, Heliopsis, Hofmeisteria*, Jaliscoa*, Jaumea, Koanophyllon, Lagascea, Lasianthaea, Melampodium, Capparis, Cleome, Crataeva, Forchhameria, Gynandropsis, Morisonia, Polanisia Mikania, Montanoa, Onoseris, Otopappus, Pachythamnus, Parthenium, Pectis, Perityle, Perymenium, Philactis, Pittocaulon*,

Caricaceae

Jacaratia, Jarilla Porophyllum, Psacalium, Roldana, Sanvitalia, Sclerocarpus, Senecio, Simsia, Sinclairia, Stevia, Steviopsis, Stuessya*, Tagetes,

Caryophyllaceae

Drymaria Tehuana*, Tetrachyron, Tithonia, Tragoceros, Tridax, Trigonospermum, Trixis, Verbesina,

Celastraceae Vernonia, Viguiera, Wedelia, Zaluzania, Zinnia

Crossopetalum, Elaeadendron, Hemiangium, Convolvulaceae Pristimera, Schaefferia, Wimmeria

Bonamia, Calycobolus, Convolvulus, 
Apéndice. Continuación.

Cuscuta, Evolvulus, Ipomoea, Jacquemontia, Jatropha, Manihot, Ophellantha, Sapium, Merremia, Operculina Sebastiania, Stillingia, Tragia

Costaceae

Costus

Crassulaceae

Echeveria, Graptopetalum, Pachyphytum*,

Sedum, Thompsonella*

Cucurbitaceae

Apatzingania*, Cayaponia, Chalema*,

Cionosicyos, Cucurbita, Cyclanthera,

Dieterlea*, Doyerea, Echinopepon,

Ibervillea, Luffa, Melothria, Microsechium, Polyclathra, Rytidostylis, Schizocarpum,

Sechiopsis, Sicydium, Sicyos

Cytinaceae

Bdallophytum

Cyperaceae

Bulbostylis, Carex, Cyperus, Fimbristylis, Kyllinga, Scleria

Dilleniaceae

Tetracera

Dioscoreaceae

Dioscorea

Ebenaceae

Diospyros

Erythroxylaceae

Erythroxylum

Euphorbiaceae

Acalypha, Adelia, Argythamnia, Bernardia, Cnidoscolus, Croton, Dalechampia,

Dalembertia, Enriquebeltrania*, Euphorbia,
Fouquieriaceae

Fouquieria

Gesneriaceae

Achimenes, Eucodonia*

Gramineae

Anthephora, Aristida, Arundinella, Axonopus, Bouteloua, Cathestecum, Cenchrus, Chaetium, Chloris, Chusquea, Ctenium, Diectomis, Digitaria, Elyonurus, Eragrostis, Eriochloa, Euclasta, Gouinia, Guadua, Heteropogon, Hilaria, Ixophorus, Lasiacis, Leptochloa, Muhlenbergia, Oplismenus, Otatea, Panicum, Paspalum, Pennisetum, Pereilema, Rhipidocladium, Schizachyrium, Setaria, Setariopsis, Sorghastrum, Sporobolus, Trachypogon, Tripsacum, Tristachya, Urochloa

Hernandiaceae

Gyrocarpus

Hydrophyllaceae

Nama, Wigandia

Iridaceae

Alophia, Cypella, Eleutherine, Nemastylis, Tigridia

Julianiaceae

Amphipterygium

Krameriaceae

Krameria

Labiatae

Asterohyptis*, Hyptis, Salvia, Stachys 
Apéndice. Continuación.

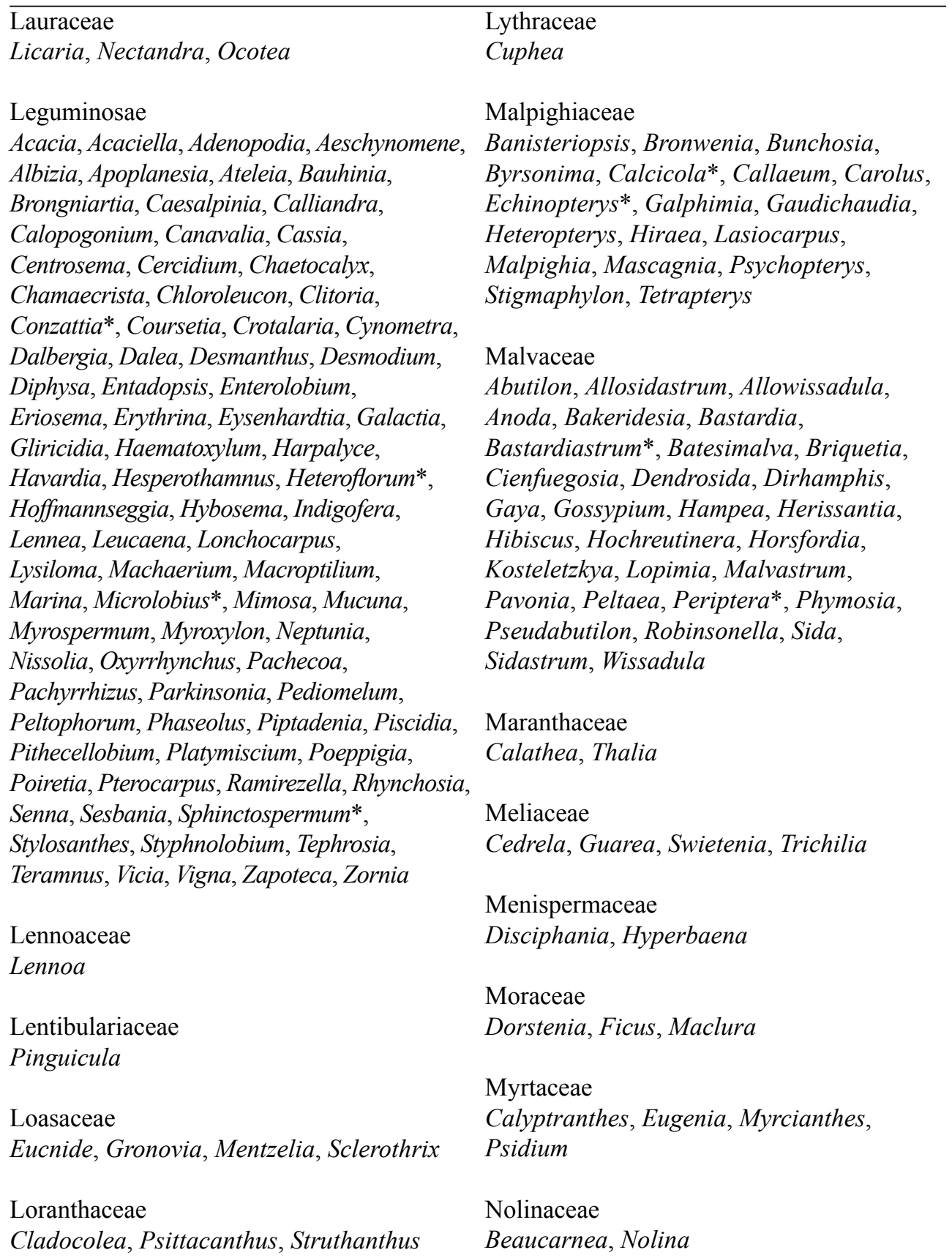


Apéndice. Continuación.

Nyctaginaceae
Boerhavia, Commicarpus, Grajalesia,
Guapira, Mirabilis, Neea, Okenia, Pisonia,
Pisoniella, Salpianthus, Torrubia

Ochnaceae

Ouratea

Olacaceae

Schoepfia, Ximenia

Oleaceae

Forestiera, Fraxinus

Onagraceae

Gongylocarpus, Hauya, Lopezia

Opiliaceae

Agonandra

Orchidaceae

Aulosepalum, Barkeria, Bletia, Brassavola,

Catasetum, Clowesia, Cyrtopodium,

Deiregyne, Dendrophylax, Dichromanthus, Encyclia, Epidendrum, Erycina*, Govenia Habenaria, Hexalactris, Leochilus,

Malaxis, Mesadenus, Mormodes, Myrmecophila, Oncidium, Physogyne*, Prostechea, Rhynchostele, Sarcoglottis, Scaphyglottis, Schiedeella, Trichocentrum, Triphora

Orobanchaceae

Buchnera, Lamourouxia, Melasma

Oxalidaceae

Oxalis

Palmae

Brahea, Sabal
Papaveraceae

Bocconia

Passifloraceae

Passiflora

Phyllanthaceae

Astrocasia, Margaritaria, Meineckia*,

Phyllanthus, Savia

Phytolaccaceae

Agdestis, Stegnosperma

Picodendraceae

Piranhea

Piperaceae

Peperomia, Piper

Picramniaceae

Alvaradoa

Plantaginaceae

Bacopa, Conobea, Russelia,

Schistophragma, Scoparia

Plocospermataceae

Plocosperma

Plumbaginaceae

Plumbago

Polemoniaceae

Bonplandia, Loeselia

Polygalaceae

Polygala, Securidaca

Polygonaceae

Antigonon, Coccoloba, Gymnopodium, Neomillspaughia, Podopterus, Ruprechtia 
Apéndice. Continuación.

Portulacaceae

Portulaca, Talinum

Pterostemonaceae

Pterostemon*

Ranunculaceae

Clematis, Thalictrum

Rhamnaceae

Colubrina, Condalia, Gouania, Karwinskia, Krugiodendron, Zizyphus

Rosaceae

Prunus

Rubiaceae

Arachnothryx, Bouvardia, Chiococca,

Chomelia, Coutaportla*, Crusea, Deppea,

Diodella, Diphragmus*, Exostema,

Glossostipula, Guettarda, Hamelia, Hedyotis, Theophrastaceae

Hintonia, Machaonia, Mitracarpus,

Paederia, Randia, Rogiera, Simira, Sommera

Rutaceae

Amyris, Casimiroa, Esenbeckia, Helietta,

Megastigma, Monniera, Pilocarpus,

Polyaster*, Stauranthus*, Zanthoxylum

Salicaceae

Casearia, Homalium, Neopringlea, Prockia, Trigoniaceae

Samyda, Xylosma

Sapindaceae

Balsas*, Cardiospermum, Cupania, Exothea, Piriqueta, Turnera

Houssayanthus, Matayba, Paullinia,

Sapindus, Serjania, Thouinia, Thouinidium,

Ungnadia, Urvillea

Sapotaceae

Sideroxylon
Scrophulariaceae

Capraria

Simaroubaceae

Castela, Recchia*

Smilacaceae

Smilax

Solanaceae

Capsicum, Cestrum, Datura, Lycianthes,

Nicotiana, Physalis, Solandra, Solanum

Spigeliaceae

Spigelia

Sterculiaceae

Ayenia, Byttneria, Guazuma, Helicteres,

Melochia, Physodium*, Waltheria

Bonellia

Thymeleaceae

Daphnopsis

Tiliaceae

Corchorus, Heliocarpus, Luehea,

Trichospermum, Triumfetta

Trigonia

Turneraceae

Ulmaceae

Celtis, Phyllostylon

Umbelliferae

Arracacia, Prionosciadium, Rhodosciadium 
Apéndice. Continuación.

\begin{tabular}{ll}
\hline Urticaceae & Viscaceae \\
Discocnide, Myriocarpa, Pilea, Pouzolzia, & Phoradendron \\
Urera, Urtica & \\
Valerianaceae & $\begin{array}{l}\text { Vitaceae } \\
\text { Ampelocissus, Ampelopsis, Cissus }\end{array}$ \\
Valeriana & Zamiaceae \\
Verbenaceae & Dioon \\
$\begin{array}{l}\text { Bouchea, Citharexylum, Lantana, Lippia, } \\
\text { Petrea, Priva, Stachytarpheta, Tamonea, }\end{array}$ & Zygophyllaceae \\
Vitex, Xolocotzia* & Guaiacum, Kallstroemia \\
Violaceae & \\
Hybanthus & \\
\hline
\end{tabular}

